\title{
Análise Prospectiva da Espécie-Chave Cultural Umbuzeiro (Spondias Tuberosa Arruda) do Semiárido Brasileiro
}

\section{Prospective Analysis of the Key Cultural Species Umbuzeiro (Spondias Tuberosa Arruda) of the Brazilian Semiarid}

\author{
Sânzia Viviane de Farias Ferreira ${ }^{1}$ \\ Ana Regina Nascimento Campos ${ }^{1}$ \\ Maria Franco Trindade Medeiros ${ }^{2}$ \\ ${ }^{1}$ Universidade Federal de Campina Grande, Campina Grande, PB, Brasil \\ ${ }^{2}$ Universidade Federal do Rio de Janeiro, Rio de Janeiro, RJ, Brasil
}

\begin{abstract}
Resumo
O bioma caatinga é caracterizado por um clima semiárido onde o umbuzeiro (Spondias tuberosa Arruda) é uma das espécies mais comuns desta paisagem. O presente trabalho teve a finalidade de fazer um estudo prospectivo do umbuzeiro. O levantamento de documentos foi realizado por palavras-chave nas bases de dados do Instituto Nacional da Propriedade Industrial, da World Intellectual Property Organization e do Derwent Innovations Index através de planilhas eletrônicas nas quais foram levadas em consideração o número de pedidos de depósito, ano, país de depósito, tipos de depositantes, a área de aplicação e o código de Classificação Internacional de Patentes. Ao todo foram resgatados 32 registros, mas após a conferência dos documentos repetidos, foi contabilizado um total de 11 depósitos entre os anos de 2010 e 2016. Foi constatado que os depósitos feitos foram, em sua maioria, realizados por pesquisadores brasileiros, na área de alimentos, categorizados nas seções de necessidades humanas, química e metalurgia.
\end{abstract}

Palavras-chave: Caatinga. Umbu. Prospecção Tecnológica.

\begin{abstract}
The Caatinga biome is characterized by a semiarid climate where the umbuzeiro (Spondias tuberosa Arruda) is one of the most common species in this landscape. The present work aimed to make a prospective study of umbuzeiro. The survey was carried out by keywords in the databases of the National Institute of Industrial Property, World Intellectual Property Organization and Derwent Innovations Index using spreadsheets that took into account the number of filing requests, year, country of filing. , filers, application area and International Patent Classification code. In all, 32 records were rescued and after the conference of the repeated documents, a total of 11 deposits were counted between 2010 and 2016. It was found that the deposits made were mostly made by Brazilian researchers, in the area of food, categorized. in the sections on human needs, chemistry and metallurgy.
\end{abstract}

Keywords: Caatinga. Umbu. Technological Prospection.

Área Tecnológica: Prospecção Tecnológica. 


\section{Introdução}

O domínio caatinga predomina no Nordeste do Brasil, ocupando o estado do Ceará, mais da metade dos estados da Bahia, Paraíba, Pernambuco, Piauí e Rio Grande do Norte e quase metade de Alagoas e Sergipe, além de pequenas porções em Minas Gerais e no Maranhão, com distribuição exclusivamente brasileira (AB SABER, 1977). O clima que o define é semiárido, caracterizado por altas temperaturas, chuvas irregulares concentradas nos primeiros meses do ano e, devido a essas disponibilidades hídricas de variação na distribuição das chuvas e restrição do período chuvoso, sua vegetação desenvolveu uma série de adaptações às condições de aridez, caracterizada por árvores e arbustos baixos com galhos retorcidos, ervas rasteiras e cactos (SAMPAIO, 1995).

Uma das espécies-chave na paisagem da caatinga é o umbuzeiro (Spondias tuberosa Arruda), que se adapta perfeitamente a períodos prolongados de seca (ARAÚJO; SANTOS, 2004). No Brasil colonial o umbuzeiro era chamado de imbu, que é uma corruptela da palavra tupi guarani "y-mb-u", que significa "árvore que dá de beber" (MEDEIROS, 2004).

O umbuzeiro pertence à família Anacardiaceae e ao gênero Spondias, que é composto por cerca de 18 espécies que ocorrem de forma espontânea ou subespontânea no Nordeste do Brasil, sendo o umbuzeiro, uma espécie exclusiva do semiárido. Planta típica do sertão e do agreste, destaca-se por possuir diversos mecanismos contra a falta de água, como as raízes modificadas, os xilopódios (ARAÚJO; SANTOS, 2004); é xerófila e caducifólia, por isso adaptada ao calor, aos solos pobres e de baixa densidade pluvial (MENDES, 1990).

Trata-se de uma espécie que pode alcançar mais de $7 \mathrm{~m}$ de altura com copa medindo até $22 \mathrm{~m}$ de diâmetro. $\mathrm{O}$ tronco é atrofiado e retorcido e as flores são brancas, agrupadas e perfumadas, servindo de alimento para abelhas. Suas raízes são compostas de órgãos de reservas denominados xilopódios ou túberas. Os frutos, conhecidos popularmente por umbu, são globulosos, apresentando coloração amarelo-esverdeado, com polpa mole e suculenta de sabor agridoce e agradável. No seu interior há um único caroço, onde se encontra a semente (SILVA; SILVA, 1976).

Através do conhecimento popular sobre a Spondias tuberosa foram realizados estudos que identificaram as diversas finalidades de tratamento de algumas patologias, como inflamações provocadas por microrganismos, diabetes, cólicas uterinas, dores de estômago e diminuição nos níveis de colesterol (LINS NETO; PERONE; ALBUQUERQUE, 2010).

O umbu se constitui, também, em fonte de renda para as famílias dos agricultores da região semiárida do Nordeste (IBGE, 2001; LIMA, 1992; SANTOS, 1998). Levantamentos socioeconômicos indicam que esta é uma atividade de grande importância em áreas expressivas do semiárido (GRANJA, 1985). Agricultores dessa região têm a prática de vender os frutos do umbu in natura, como também processado, na forma de doces, geleias, sorvetes e, ainda, em misturas como a "umbuzada".

No entanto, é um fruto de rápida sazonalidade, apresentando um período de safra curto e alta perecibilidade em condições naturais (DUQUE, 1980). Neste sentido, é importante o desenvolvimento de pesquisas tecnológicas que possam auxiliar o processamento destes frutos $e$, ainda, aumentar seu tempo de prateleira, influenciando de forma significativa a indústria e a sociedade como um todo. Tais pesquisas devem ocorrer de forma contínua através de metodo- 
logias de prospecção tecnológica, realizadas em bancos de dados valiosos e a partir de fontes confiáveis (AMPARO et al., 2012) para a obtenção de informações e tomadas de decisões.

Assim, tomando este viés da prospecção tecnológica, o presente trabalho teve a finalidade de fazer um estudo prospectivo do umbuzeiro, a fim de analisar as tecnologias e inovações associadas a esta espécie através de um mapeamento dos pedidos de depósito de patente, onde foi levado em consideração o número de pedidos de depósito, ano, país de depósito, tipos de depositantes, a área de aplicação e o código de Classificação Internacional de Patentes.

\section{Metodologia}

O mapeamento de pedidos de depósitos de patente acerca da prospecção tecnológica do umbuzeiro teve início a partir de pesquisas nas bases de dados Instituto Nacional da Propriedade Industrial do Brasil (INPI), World Intellectual Property Organization (WIPO) e Derwent Innovations Index (Web of Science/Clarivate Analytics). A busca foi realizada no período de agosto a outubro de 2018. Os descritores de busca foram determinados e tiveram como base as seguintes palavras-chave: "umbu", "umbuzeiro" ou "Spondias tuberosa". Não houve restrição de busca por período ou língua de publicação, tendo sido selecionados todos os documentos que faziam referência à tecnologia protegida do umbuzeiro.

Todos os pedidos de depósito de patente encontrados foram analisados e tabulados em planilha eletrônica levando-se em consideração o número de pedidos de depósito, ano, país de depósito, tipos de depositantes, as áreas de aplicação e o código de Classificação Internacional de Patentes (CIP).

\section{Resultados e Discussão}

Foram levantados 32 registros de pedido de depósito de patente (Tabela 1) que, após a eliminação dos documentos repetidos, resultaram em um total de 11 registros que tratavam da tecnologia e inovação envolvendo o umbuzeiro, para que assim fosse verificada a produção do conhecimento científico desta espécie.

Tabela 1 - Resultados da busca por pedidos de depósito de patente depositados nas bases de dados INPI, WIPO e Derwent, por palavras-chave, em 2018

\begin{tabular}{cccc} 
Palavra-chave & INPI & WIPO & DERWENT \\
Umbu & 7 & 11 & 6 \\
Umbuzeiro & 0 & 0 & 0 \\
Spondias tuberosa & 1 & 4 & 3 \\
Total & 8 & 15 & 9 \\
\hline
\end{tabular}

Fonte: Bases de dados INPI, WIPO e Derwent (2018)

Dos pedidos de depósito de patente encontrados, foram observados registros entre os anos de 2010 e 2016 (Figura 1). Ao se considerar este intervalo de tempo em sua integralidade, verificou-se um aumento no número de patentes relacionadas ao umbuzeiro. No entanto, ao se 
fazer uma análise a partir da data inicial de 2010 até o ano de 2013, pôde-se constatar dois comportamentos. O primeiro foi à ausência de depósitos em 2011. Já no segundo cenário, pôde-se observar um comportamento uniforme entre os anos de 2010, 2012 e 2013, quando houve o registro de uma patente para cada um destes anos, respectivamente. O registro de depósito de patente inicial para o umbuzeiro, ocorrido no ano de 2010, está vinculado ao processamento da matéria-prima desta espécie vegetal. Adiante, verificou-se que nos anos de 2014 e 2015 houve um novo aumento no número de patentes depositadas. Fechando o período com registros de patente para o umbuzeiro, o ano de 2016 foi o que apresentou maior número de pedidos de depósitos (quatro registros no total).

Desta forma, o comportamento ascendente nos pedidos de depósito de patente observado para o umbuzeiro pode ser o indicativo do resultado de um maior investimento financeiro em biotecnologia por parte dos países investidores na intenção de proporcionar avanços e desenvolvimento de tecnologias na área de interesse (SCHUMPETER, 1982).

Figura 1 - Evolução anual dos pedidos de depósito de patente referentes ao umbuzeiro (Spondias tuberosa Arruda), considerando o período de 2010 a 2016

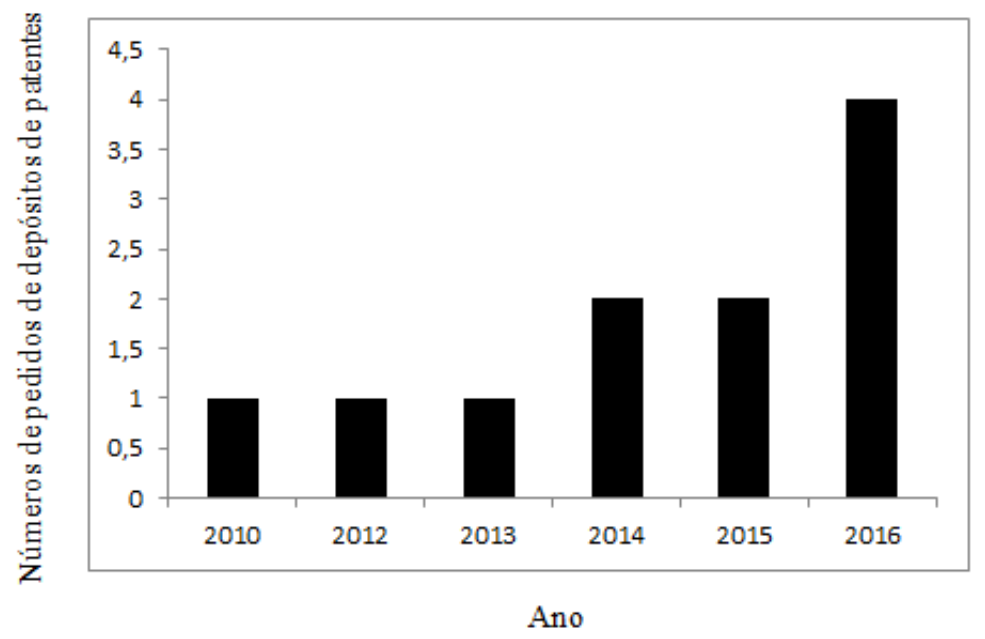

Fonte: Bases de dados INPI, WIPO e Derwent (2018)

Consideramos que, com o aumento do comércio internacional e com a crescente necessidade de proteger e incentivar os investimentos em novos produtos e métodos de fabricação, se fizeram necessárias algumas normas mínimas para os procedimentos de patenteamento e proteção à Propriedade Industrial no mundo, através de acordos multilaterais que foram firmados, tais como a Convenção de Paris, a criação da OMPI e o Tratado de Cooperação em Matéria de Patente.

Neste sentido, todas as patentes depositadas relacionadas ao umbuzeiro são oriundas do Brasil. Contudo, existem 18,2\% dos registros que são patentes depositadas no Patent Cooperation Treaty (PCT), um tratado internacional que oficializa um sistema para o depósito de pedidos de patente e que permite que se obtenham patentes em diversos países a partir de um único pedido, cujo objetivo principal é facilitar e reduzir os custos iniciais nos procedimentos nos pedidos de patentes nos países membros.

Os depositantes pertencem às Universidades Federais, Estaduais ou Institutos Federais (ICT - Instituições Científicas e Tecnológicas) brasileiros, destacando-se uma parceria com a Universidade de Genebra; e também foi observada a existência de um depositante independen- 
te. Esta realidade acerca da pesquisa tecnológica e inovação em torno do umbuzeiro reflete o que foi constatado por Garnica et al. (2006), os quais afirmam que o momento atual é de uma adequação das estruturas acadêmicas que visam ao aperfeiçoamento da gestão tecnológica para o aproveitamento dos resultados de pesquisa acadêmica. Este processo de adequação vai justamente se refletir na ação dos centros de produção do conhecimento em prol da proteção das novas tecnologias desenvolvidas em seus espaços acadêmicos.

Além disso, outra tendência observada dentre as informações coletadas na prospecção tecnológica foi o fato de que a maioria dos inventores realizou depósitos de patentes em parceria, ou seja, das 11 patentes analisadas, sete $(63,63 \%)$ foram desenvolvidas por dois ou mais inventores.

As áreas de conhecimento identificadas no estudo prospectivo do umbuzeiro mostram que a maioria dos pedidos de depósito de patente depositados está relacionada à área de alimentos (54,54\% do total de registros), seguida da área química (36,36\%) e da biotecnológica (9,10\%). Entre os pedidos de patentes depositados tiveram destaque os da tecnologia na área da Indústria de Alimentos que, conforme Santini et al. (2005), inclui o aumento da produtividade e ao mesmo tempo a redução dos custos, aumentando a competitividade no preço final do produto.

A Classificação Internacional de Patentes dispõe os documentos de patentes publicados em áreas tecnológicas a que pertencem (SERAFINI et al., 2012). Neste sentido, as 11 patentes levantadas foram categorisadas em duas seções ( $\mathrm{A}$ - necessidades humanas e $\mathrm{C}$ - química, metalurgia) e em oito classes, de acordo com a sua aplicação. Dentre os documentos analisados, a seção A se destacou com $71,42 \%$ dos pedidos de depósito de patente. A seção A englobou seis classes e a $\mathrm{C}$ duas classes, sendo estas as classes A23B, A23G, A23L, A47J, A61K, A61P, $\mathrm{C} 07 \mathrm{C}$ e $\mathrm{C} 07 \mathrm{H}$.

A classe com maior número de depósitos foi a A23L, com 23,80\%, representando a área de alimentos, na qual o umbuzeiro se destaca pela possibilidade de ser aproveitado de diversas formas, tanto para a alimentação humana, quanto para a suplementação alimentar de animais (CAMPOS, 1986, 1988, 1997, 1998; CAVALCANTI et al., 2000). Em seguida, houve destaque para a Classe $\mathrm{A} 61 \mathrm{~K}$, que se refere à preparação para finalidades médicas, odontológicas ou de higiene (19,04\%), como por exemplo, diversos usos para o tratamento de algumas patologias, como o diabetes, as inflamações, cólicas uterinas e dores de estômago (LINS NETO; PERONI; ALBUQUERQUE, 2010). Na sequência, ficaram a classe C07C, que se refere à química orgânica de compostos cíclicos e acíclicos (19,04\%) e a classe A61P, que diz respeito à atividades terapêuticas específicas de compostos químicos ou preparações medicinais (14,29\%), em que os fins terapêuticos podem estar relacionados com os constituintes presentes nesta espécie. Os frutos do umbuzeiro apresentaram pronunciada atividade antioxidante e sequestro de radicais livres, pela presença de compostos fenólicos e vitamina $\mathrm{C}$, bem como há relatos da presença de flavonoides, antocianinas e carotenoides. Quando avaliada a presença de compostos fenólicos de baixo peso molecular, ou fenólicos simples por Ultra Fast Liquid Chromatograph (UFLC), constatou-se a presença de ácido gálico, clorogênico, protocatecuico, p-cumárico, vanílico e ferúlico, bem conhecidos por suas propriedades terapêuticas. O componente fenólico majoritário foi o ácido clorogênico (SILVA et al., 2014). Após, estiveram posicionadas em termos quantitativos de patentes, a classe $\mathrm{C} 07 \mathrm{H}$, que se refere a química orgânica de açúcares e seus derivados $(9,52 \%)$, e a classe A23B, que se refere à conservação, amadurecimento químico de frutas, legumes ou enlatados $(4,77 \%)$. Estas revelam como é importante a correta determina- 
ção do estado de maturação em que um fruto se encontra, essencial para que a colheita seja efetuada no momento certo (KLUGE et al., 2002) e, também, para que desta forma se tenham subsídios técnicos que visem à ampliação do tempo de armazenamento sem, contudo, alterar suas características físicas, organolépticas e nutricionais (ABREU et al., 1998). Depois esteve a classe A23G, que se refere ao cacau e seus derivados $(4,77 \%)$ e, por fim, A47J, que se refere à equipamentos de cozinha ou aparelhos para fazer bebidas $(4,77 \%)$.

Já o detalhamento obtido através da busca das subdivisões de patentes em grupos e subgrupos apontou para um total de 26 tipologias diferentes (Tabela 2).

Tabela 2 - Resultados da Classificação Internacional dos pedidos de depósito de patente referentes ao umbuzeiro nas bases de dados INPI, WIPO e Derwent, por palavras-chave, em 2018

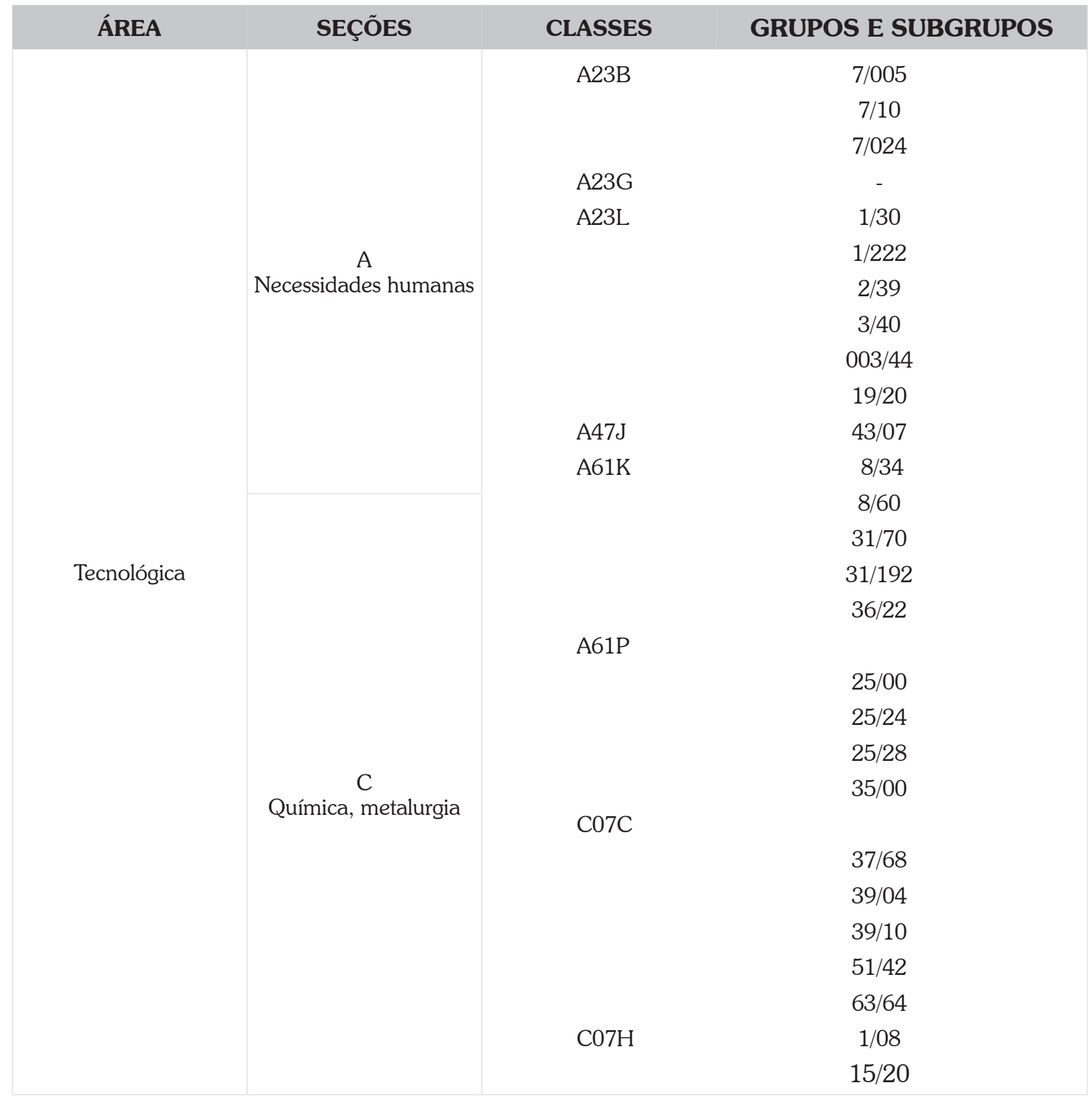

Fonte: Bases de dados INPI, WIPO e Derwent (2018) 
A classe A23B esteve relacionada aos grupos A23B 7/005 com adição de produtos químicos; A23B 7/10 conservação com ácido, fermentação ácida e A23B 7/024 secagem por congelamento (33,33\% dos registros para cada grupo citado).

E para a classe A23G, não foi identificado nenhum grupo. Este detalhamento pode proporcionar uma avaliação das aplicações e pode servir como um indicativo para que se busque um melhor direcionamento de futuros estudos.

Assim, para a classe A23L foram identificados os grupos A23L 1/30 contendo aditivos; A23L 1/222 frutas; A23L 2/39, que se refere a composições secas; A23L 3/40 seleção ou secagem em estufa; A23L 19/20 pela conservação em salmoura ou vinagre (14,29\% dos registros para cada grupo citado); e A23L 003/44 secagem por congelamento (28,55\%).

Já a classe A47J teve o grupo A47J 43/07 peças, detalhes ou instrumentos para bater $(100 \%$ dos registros). Para a classe A61K foram identificados os grupos A61K 8/34 álcoois; A61K 8/60 contendo grupos perfluor; A61K 31/70 carboidratos e açúcares; A61K 31/192 tendo grupos aromáticos (com 12,5\% dos registros para cada grupo citado); e A61K 36/22 Anacardiaceae (família do Sumaque) (50\% dos registros).

Em A61P foram encontrados os grupos A61P 25/00 fármacos para o tratamento de doenças do sistema nervoso; A61P 25/24 antidepressivos; A61P 25/28 para o tratamento da doença de Alzheimer ou outras formas de demência (com 20\% dos registros para cada grupo citado); e A61P 35/00 agentes antineoplásicos (40\% dos registros).

Na classe C07C estiveram presentes os grupos C07C 37/68 separação, purificação, estabilização e uso de aditivos; C07C 39/04 fenol; C07C 39/10 poliidroxibenzenos; C07C 51/42 separação, purificação e estabilização; e C07C 63/64 ácidos monocíclicos com insaturação fora do anel aromático (com $20 \%$ dos registros para cada grupo citado).

Por fim, na classe $\mathrm{C} 07 \mathrm{H}$, os grupos registrados foram $\mathrm{C} 07 \mathrm{H} 1 / 08$ a partir de produtos naturais (33,33\% dos registros) e C07H 15/20 anéis carbocíclicos (66,67\%).

\section{Considerações Finais}

A prospecção tecnológica permite analisar os conhecimentos e tecnologias já produzidos sobre determinado produto, servindo de orientação geral para a prática de pesquisa científica quando se busca a inovação de produtos que garantam um retorno financeiro para os investidores. Neste sentido, e com base na análise dos dados da prospecção tecnológica do umbuzeiro, foi verificado que os 11 depósitos feitos foram realizados em grande parte por pesquisadores brasileiros pertencentes a Universidades Federais, Estaduais ou Institutos Federais, além de um depositante independente.

Os registros indicam ter havido produção e proteção de tecnologia e inovação em um intervalo de menos de uma década, entre os anos de 2010 e 2016. Estes pedidos de patente apresentaram processos e produtos comercializáveis dentro das áreas de alimento onde existe uma grande variedade de produtos à base de umbu, a exemplo da umbuzada, geleias, compotas, sorvetes, polpas congeladas, dentre outros produtos, seguida da área química e da área de biotecnologia para que assim se diminua o grande desperdício da fruta in natura. Tais resultados foram categorizados em seções (A e C) e em oito classes de acordo com a sua aplicação (A23B, A23G, A23L, A47J, A61K, A61P, C07C e C07H). Sendo assim, a coleta e a comercialização dos 
frutos dentro da área de alimentos é uma atividade cultural passada de geração a geração com base alimentar e econômica para a complementação da renda nas áreas rurais dos agricultores do semiárido.

Embora tenha sido constatado esse cenário voltado à produção e proteção de processos e produtos, pode-se afirmar que ainda é pequeno o número de pedidos de depósito de patente referentes ao umbuzeiro. Desta forma, fica evidente a necessidade de se realizar pesquisas sobre esta espécie endêmica da caatinga brasileira e de importância para a população local. Será através desse movimento de ampliação das pesquisas que novas tecnologias voltadas à produção e ao beneficiamento do umbuzeiro poderão surgir e ser protegidas por patentes concedidas. É então esse processo de produção e inovação tecnológica que será capaz de gerar um retorno às pessoas inseridas no contexto do semiárido, ao se apresentar a elas possibilidades de melhoria na qualidade de vida através das inovações tecnológicas.

\section{Referências}

AB'SABER, A.N. Os domínios morfoclimáticos na América do Sul: primeira aproximação.

Geomorfologia, [S.1.], v. 53, p. 1-23, 1977.

ABREU, C. M. P.; CARVALHO, V. D. de; GONÇALVES, N. B. Cuidados pós-colheita e qualidade do abacaxi para exportação. Informe Agropecuário, Belo Horizonte, v.19, n.195, p. 70-72, 1998.

AMPARO, K. K. S. et al. Estudo de caso utilizando mapeamento de prospecção tecnológica como principal ferramenta de busca científica. Perspectivas em Ciência da Informação, [S.I.], v. 17, n. 4, p. 195-209, 2012.

ARAÚJO, F. P. de; SANTOS, C. A. F. Substituição de copa do umbuzeiro por algumas espécies do gênero Spondias. In: REUNIÃO NORDESTINA DE BOTÂNICA, 28. Petrolina. Petrolina. [Anais...]. Petrolina: SBB; Embrapa, 2004.

BRASIL. Lei no 9.279, de 14 de maio de 1996. Regula direitos e obrigações relativos à propriedade industrial. Diário Oficial da União, 15 de maio de 1996, p. 8353. Disponível em: http://www. planalto.gov.br/ccivil_03/leis/L9279.htm. Acesso em: 14 abr. 2019.

CAMPOS, C. O. Estudos de Quebra de Dormência da Semente do Umbuzeiro (Spondias tuberosa Arr. Câmara).1986. 71f. (Mestrado em Fitotecnia) - Universidade Federal do Ceará, Fortaleza, 1986.

CAMPOS, C. O. Industrialização caseira do umbu. Uma nova perspectiva para o semi-árido. Salvador: EPABA (EBDA). 1988. 20p. (Circular Técnica, 14).

CAMPOS, C. O.; SOUZA, E. G.; MENEZES FILHO, D. S. Domesticação do umbuzeiro, com vistas ao aproveitamento industrial. Juazeiro, BA. UNEB / FAMESF, EBDA. 1998. 34p. il. (Relatório para o CNPq).

CAMPOS, C.O.; SOUZA, E. G.; MENEZES, D.S.; CAMPOS, N. S. F. Umbu, mais algumas considerações. In.: CONGRESSO DAS UNIVERSIDADES ESTADUAIS DA BAHIA, I, 1997 , Salvador, BA. Resumos [...] Salvador, BA. UNEB. 1997.

CAVAlCANTI, N de B.; RESENDE, G. M. de; BRITO, L. T. de L. Processamento do fruto do imbuzeiro (Spondias tuberosa Arr. Cam.). Ciência e Agrotecnologia, Lavras, v. 24, n. 1, p. 252259. 2000. 
DUQUE, J. G. O umbuzeiro. In: O nordeste e as lavouras xerófilas. 3. ed. Mossoró: Fundação Guimarães Duque, 1980. p. 283-289.

GARNICA, L. A.; OLIVEIRA, R. M.; TORKOMIAN, A. L. V. Propriedade intelectual e titularidade de patentes universitárias: um estudo piloto na Universidade Federal de São Carlos - UFSCar. In: SIMPÓSIO DE GESTÃO DA INOVAÇÃO TECNOLÓGICA, 24, 2004, Anais [...] Gramado. Gramado: Associação Nacional de Pós-Graduação e Pesquisa em Administração, 2006.

GRANJA, M.L.B.B. Efeito de métodos de preservação e tempo de estocagem na qualidade dos sucos simples de umbu (Spondias tuberosa Arruda Câmara) e mangaba (Mancornia spenciosa Muell.). 1985. 102f. Dissertação (Mestrado) - Universidade Federal da Paraíba, João Pessoa, 1985.

IBGE. Sistema IBGE de Recuperação Automática - SIDRA. Produção Extrativa Vegetal Quantidade produzida na extração vegetal - Umbu (fruto) (Tonelada) Ano. 1990 a 2001. Banco de Dados Agregados. Disponível em: www.sidra.ibge.gov.br/bda/. Acesso em: 15 nov. 2018.

KLUGE, R. A.; NACHTIGAL, J. C.; BILHALVA, A. B. Fisiologia e manejo pós-colheita de frutas de clima temperado. Pelotas: UFPel. 2002. 163p.

KUPFER, D; TIGRE P. Modelo SENAI de Prospecção: Documento Metodológico. Capítulo 2:

Prospecção Tecnológica. In: ORGANIZACION INTERNACIONAL DEL TRABAJO CINTERFOR. Papeles de La Oficina Técnica n. 14, Montevideo: OIT/CINTERFER; 2004. Anais [...], Montevideo, 2004.

LIMA, M. de F. M. de. Desidratação de polpa de umbu em leito de jorro: Estudos fluidodinâmicos e térmicos. 1992. 118f. Dissertação (Mestrado em Engenharia Agrícola). Departamento de engenharia Agrícola. Universidade Federal da Paraíba. Campina Grande, PB, 1992.

LINS NETO, E. M. F; PERONI, N.; ALBUQUERQUE, U. P. Traditional Knowledge and Management of Umbu (Spondias tuberosa, Anacardiaceae): an endemic Species from the Semi-Arid Region of Northeastern Brazil. Economic Botany, v. 64, n. 1, p. 11-21, 2010.

MEDEIROS, S. S. A. Obtenção de pó de umbu (Spondias tuberosa Arr. Câmara) para umbuzada: processamento e caracterização do pó. 2004. 102f. Dissertação (Mestrado em Engenharia Agrícola). Departamento de engenharia Agrícola. Universidade Federal de Campina Grande. Campina Grande, 2004.

MENDES, B. V. Umbuzeiro (Spondias tuberos Arr. Cam.): importante fruteira do semi-árido. Mossoró. ESAM, 1990. 66p. (Coleção Mossoroense, Série C).

SAMPAIO, E. V. S. B. Overview of the Brazilian caatinga. In: BULLOCK, S. H.; MOONEY, H. A.; MEDINA, E. (ed.). Seasonally dry tropical forest. Cambridge: Cambridge University Press, 1995. p. 35-63.

SANTINI, G. A.; SCHIAVI, S. M. A.; SOUZA FILHO, H. M. Inovações tecnológicas em cadeias agroindustriais: alguns casos do segmento de processamento de carnes, leite e café no Brasil. In: XII SIMPEP - BAURU, novembro 2005.

SANTOS, C. A. F. Relação entre caracteres de produção do umbuzeiro com características e teor de água do solo. Revista Brasileira de Fruticultura, Cruz das almas, v. 10, n. 2, p. 206- 212, ago. 1998. 
SCHUMPETER, J. A. A teoria do desenvolvimento econômico: uma investigação sobre lucros, capital, crédito, juro e o ciclo econômico. São Paulo: Abril Cultural, 169 p., 1982.

SERAFINI, M. R.; QUINTANS, J. S. S.; ANTONIOLLI, A. R.; SANTOS, M. R. V.; QUINTANS JUNIOR, L. J. Mapeamento de tecnologias patenteáveis com o uso da hecogenina. Revista Geintec, v. 2, n. 5, p. 427-435, 2012.

SILVA, A. Q. da ; SILVA, M. A. da G. O. Observações morfológicas e Fisiologia sobre Spondias tuberosa, Arr. Câmara. In: Anais [...] CONGRESSO NACIONAL DE BOTÂNICA, 25 Mossoró, RN. 1974. Rio de Janeiro, Sociedade Botânica do Brasil, 1976. p. 5-15.

SILVA, G. A.; BRITO, N. J. N.; SANTOS, E. C. G.; LÓPEZ, J. A.; ALMEIDA, M. G. Gênero Spondias: Aspectos botânicos, composição química e potencial farmacológico. Revista de Biologia e Farmácia, v. 10, n. 1, 2014.

\section{Sobre os Autores}

\section{Sânzia Viviane de Farias Ferreira}

E-mail: sanziafarias@gmail.com

Bacharelado e Licenciatura plena em Ciências Biológicas pela UEPB (2003); Licenciatura em Ciências Sociais, pela UFCG (2002). Pós-graduação Lato Sensu (especialização) em Fundamentos da Educação: Práticas Pedagógicas interdisciplinares pela UEPB (2014). É aluna no Programa de Pós-Graduação em Ciências Naturais e Biotecnologia da UFCG (PPG-CNBiotec/UFCG).

Endereço profissional: Universidade Federal de Campina Grande, PPG em Ciências Naturais e Biotecnologia PPGCNBiotec. Rua 15 de Novembro, n. 723, Bairro Basílio da Fonseca, Cuité, Paraíba. CEP: 58.175.000.

\section{Ana Regina Nascimento Campos}

E-mail: arncampos@yahoo.com.br

Graduação em Química Industrial (2000), Mestrado em Engenharia Química (2003) e Doutorado em Engenharia de Processos (2008).

Endereço profissional: Universidade Federal de Campina Grande, Centro de Ciências e Tecnologia/Unidade Acadêmica de Engenharia Química. Rua Aprígio Veloso, n. 882, Universitário, Campina Grande, Paraíba. CEP: 58.428-830.

\section{Maria Franco Trindade Medeiros}

E-mail:mariaftm@hotmail.com

Bacharelado e Licenciatura em Ciências Biológicas e Ambientais pela Universidade Santa Úrsula (2000), Mestrado e Doutorado em Ciências Biológicas (Botânica) pelo Museu Nacional da UFRJ (2003 e 2007, respectivamente); e Pós-doutorados pelos Programas de Pós-Graduação em Botânica e em Ecologia, ambos do Departamento de Biologia da UFRPE (2011 e 2012, respectivamente).

Endereço profissional: Museu Nacional da Universidade Federal do Rio de Janeiro - MN/UFRJ. Departamento de Botânica, Laboratório de Etnobotânica e Taxonomia de Angiospermas. Rua Quinta da Boa Vista, s/n, São Cristóvão, Rio de Janeiro. CEP: 20.940-040. 